PHARMACY PRACTICE

\title{
Practice Spotlight: Dawn Dalen
}

\author{
Dawn Dalen, BSP, ACPR, PharmD \\ Interior Health Authority \\ Kelowna General Hospital - \\ Pharmacy Services \\ Kelowna, British Columbia
}

$\mathrm{I}_{\mathrm{H}}^{\mathrm{s}}$ 's 9 AM on a Thursday morning at Kelowna General Hospital. The emergency pharmacist enters the Emergency Department, which is bursting at the seams. There are patients in the hallways, the trauma bays are full, and there is a long list of patients requiring assessment by a pharmacist. This scenario is not unique to the Kelowna General Hospital: it is played out every day in emergency departments across the country.

The provision of clinical pharmacy services in the emergency department was first reported in 1977, ${ }^{1}$ but economic constraints have generally precluded emergency departments from having their own dedicated clinical pharmacists. $^{2}$ In a recent Canadian prospective observational study, $12.0 \%$ (95\% confidence interval [CI] 10.1\%-14.2\%) of emergency department visits were drug-related, and $68.0 \%$ of these visits (95\% CI 59.0\%-76.2\%) were deemed preventable. ${ }^{3}$ Furthermore, patients visiting the emergency department because of drug-related problems were more likely to be admitted to hospital and had a longer length of stay than those visiting the emergency department for nondrug reasons. ${ }^{3}$ In an economic analysis conducted at one of the larger emergency departments in the United States, between $\$ 4.68$ and $\$ 16.70$ was saved for every dollar spent on a pharmacist. ${ }^{4}$ Therefore, because of the enormous economic burden associated with adverse drug events, it may be highly cost-effective to use the high-level expertise of a clinical pharmacist in the emergency department. $^{2}$

The Kelowna General Hospital is a 344-bed tertiary care facility serving about 300000 people in the Okanagan Valley. It is a trauma and referral hospital for the Interior of British Columbia. The Emergency Department is a 23-bed unit, with 2 trauma rooms and a seclusion room. The department serves about 54000 patients per year, which makes it the busiest emergency department in the BC Interior. With recent regional restructuring, the number of patients with high acuity scores seen in the emergency department has increased by $225 \%$ over the past 5 years. In response to this growth, a new Emergency Department with 46 beds is due to replace the current department in 2012.

Dawn Dalen began working in the Kelowna General Hospital Emergency Department in 2005, after completing her PharmD at the University of British Columbia. It was the first time a clinical pharmacist had been dedicated to the department, despite requests for such a position over several years. Before she started in the department, Dr Dalen met with the key emergency physicians and allied health care professionals to discuss the role of the emergency pharmacist and also to obtain their input on how a clinical pharmacist could be integrated into the department.

The clinical pharmacist works Monday to Friday and is responsible for providing care to all patients in the Emergency Department, both those who have been admitted and are being boarded in the department and those who are being treated as outpatients. Pharmaceutical care in the emergency setting presents unique challenges not seen in other areas of the hospital, given the high patient volume, rapid turnover, and diversity of cases. The patient's history is often not completed before the pharmacist does her assessment; furthermore, such histories may be difficult to obtain in the Emergency Department because of the clinical situation. The Emergency Department is an area at especially high risk for medication errors, since common safety mechanisms used elsewhere in the hospital are not available, including prospective medication order review, and preparation and dispensing of medications in the pharmacy. Medication orders are often given verbally, and the drugs are prepared and administered to the patient right at the point of care. The clinical pharmacist is constantly prioritizing who to spend time with, trying to focus on patients with chronic conditions who are taking many medications, a situation in which pharmacists are known to have an impact on outcomes. Patients needing assessment by the clinical pharmacist are identified by chief complaint, acuity, complicated or unique pharmacotherapy, and/or referral from another health care professional. In addition, the pharmacist assists with drug therapy for advanced cardiac life support and with management of drug therapy during medical emergencies. Patients with acute venous thromboembolism return to the 
Emergency Department daily to be seen by the pharmacist until the transition to warfarin therapy has been safely accomplished and their care can be transferred back to the primary care provider. Providing discharge medication teaching, undertaking seamless care activities, and answering many therapeutic queries posed by other health care professionals are other activities that may occupy the emergency pharmacist's time.

Education is a significant component of the pharmacist's role in the emergency setting. Formal presentations, in-service education, and informal education are all provided to other team members, residents, and students. There are many opportunities to educate others about the efficacy and toxicity of pharmacotherapy, medication safety, and methods of administering, titrating, and monitoring agents.

The emergency pharmacist is a local, regional, and provincial resource. Nonclinical activities include collaborating with other health care professionals to develop, manage, and assist in implementing pharmacotherapeutic protocols or programs to improve appropriate drug use or quality of patient care. Time is also spent conducting drug-use evaluation and practice-based research when clinical questions arise. The emergency pharmacist has become a key team member for all major provincial and regional quality improvement initiatives that involve pharmacotherapy and continues to represent the hospital's Pharmacy Department on the regional Emergency Department Coordinating Committee for the Interior Health Authority and the Emergency Department Advisory Committee within Kelowna General Hospital. The time spent on any given activity may vary from day to day, depending on the workload within the Emergency Department.

Given the potential impact of clinical pharmacists in the emergency setting, many departments have implemented or are assessing the feasibility of extending clinical pharmacist hours. The use of pharmacy technicians is also being considered to assist with medication reconciliation. The emergency department is a key transition point for patients entering acute care and being discharged back to the community, and discrepancies in medication therapy are common. The number of patients seen in the Emergency Department of the Kelowna General Hospital is increasing every year, primarily because of the wave of aging baby boomers and the increased number of people relying on the emergency department for their primary care needs. The role of the pharmacist is paramount to improving rational, safe, cost-effective medication therapy within emergency departments and should be considered the standard of care for this vulnerable patient population.

References

1. Elenbaas R, Waeckerle J, McNabney WK. The clinical pharmacist in emergency medicine. Am J Hosp Pharm 1977;34(8):843-846.

2. Peth HA Jr. Medication errors in the emergency department: a systems approach to minimizing risk. Emerg Med Clin North Am 2003;21(1): 141-158.

3. Zed PJ, Abu-Laban RB, Balen RM, Loewen PS, Hohl CM, Brubacher JR, et al. Incidence, severity and preventability of medication-related visits to the emergency department: a prospective study. CMAJ 2008;178(12): 1563-1569.

4. Delgrado G Jr. Emergency department pharmacy practice: another primary care opportunity [presentation]. ASHP Midyear Clinical Meeting; 2004 Dec 6; Orlando (FL).

The Practice Spotlight series highlights the accomplishments of Canadian pharmacists with unique practices in hospitals and related health care settings. If you have a unique or innovative practice, or you know someone else who should be profiled, please submit your contact information to Mary Ensom, Editor of CJHP (cjhpedit@cshp.ca), and one of our Associate Editors will be in touch with you. 